Technological University Dublin

DÜBLIN

ARROW@TU Dublin

2018

\title{
A Physical Biology, the Electron Neutrino Mass, and the role of Quantum Mechanics in Nature
}

Maurice Goodman

Technological University Dublin, maurice.goodman@tudublin.ie

Follow this and additional works at: https://arrow.tudublin.ie/scschphyart

Part of the Biology Commons, and the Cell Biology Commons

\section{Recommended Citation}

Goodman, M. (2018)A Physical Biology, the Electron Neutrino Mass, and the role of Quantum Mechanics in Nature, NeuroQuantology 2018; 16(11):49-54, DOI:10.14704/nq.2018.16.11.1865

This Article is brought to you for free and open access by the School of Physics \& Clinical \& Optometric Science at ARROW@TU Dublin. It has been accepted for inclusion in Articles by an authorized administrator of ARROW@TU Dublin. For more information, please contact arrow.admin@tudublin.ie, aisling.coyne@tudublin.ie, gerard.connolly@tudublin.ie.

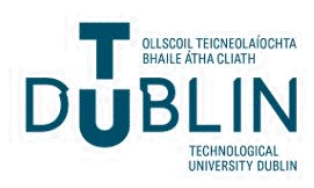




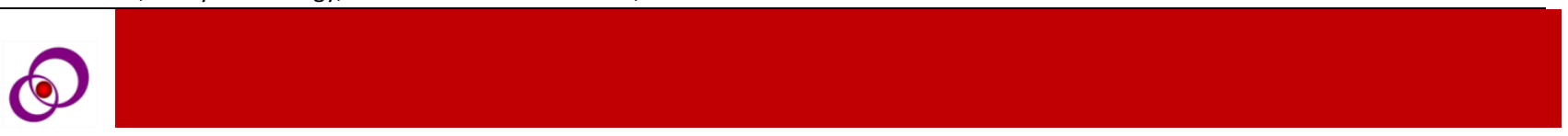

\title{
A Physical Biology, the Electron Neutrino Mass, and the role of Quantum Mechanics in Nature
}

\begin{abstract}
Maurice Goodman
ABSTRACT

In science we need to remove physics and earth science from the fundamental natural sciences and treat Biology as a fundamental natural (physical) science. Attempts to keep Biology autonomous because it is holistic, from the physical sciences, are just disguised anthropocentrism. Physical sciences have holistic features also. The autonomy of Biology is at odds with a holistic, integrated science and is preventing progress in science. For example, every cell needs a 'global' communication system to keep order and stability with rapid information transfer across cellular scales. We have yet to figure out how this is achieved. Over the last 30 years, we have understood that quantum mechanics is about information, mostly. However, we do not have a clear understanding of the physical significance of quantum mechanics in nature. Also, the view that quantum mechanics is restricted to the atomic and molecular scale is mistaken and a direct result of the mass of the electron being so big. In 1988 the mass of the electron neutrino was predicted to lie between 0.5 and $0.05 \mathrm{eV} / \mathrm{c}^{2}$ and to have a key role in Biology. This would allow quantum mechanical processes on a cellular and intercellular scale and provide a possible basis for a 'global' information system in the cell and an understanding of the information role of quantum mechanics in nature. Recent non-results, on the electron neutrino mass, from the KATRIN experiment are pushing the upper limit of the electron neutrino mass to less than $0.5 \mathrm{eV} / \mathrm{c}^{2}$ making the prediction of 30 years ago more likely.
\end{abstract}

Key Words: Natural Science, Holism, Biological Cell, Quantum Communication, Quantum Information, Electron Neutrino

DOI Number: 10.14704/nq.2018.16.11.1865 NeuroQuantology 2018; 16(11):49-54

\section{Introduction}

In 1994 a proposal for a new arrangement of the fundamental natural sciences was published (Goodman, 1994; 1997). This new arrangement required that both earth science and physics be removed as fundamental natural sciences to make progress in Biology/Materials Science and Astronomy. For reasons discussed previously, (Goodman, 2016) and (Goodman, 2017) earth science is not fundamental and should be treated as an interdisciplinary sub-field of all those that are (Astronomy, Biology, Chemistry and Nuclear Science). Physics needs to be removed from the fundamental natural sciences as it is not natural, but man-made. All the other fundamental natural sciences refer to a specific structure found in nature. Physics does not. Physics is a collection of the laws that govern the interactions and properties of all matter and energy in general and represents what we know so far about nature but not necessarily all there is to know. Finally, the new arrangement also required Biology to be treated as a physical science to make progress with a fundamental physics of biology and the cell with the goal of fully explaining the cell and, in the distant future, explaining the mind and consciousness. This paper examines the implications of these changes for Biology and our 
understanding of the role of quantum mechanics in nature.

\section{Biology is not autonomous}

If mankind were a rational species the heliocentric suggestion of Copernicus, and its subsequent confirmation, should have finished off Anthropocentrism: the belief that life and specifically man was central or most significant in the universe. The sad truth is that we are not rational and have to continually struggle to be so. Over the centuries Anthropocentrism has constantly been shown to be wrong. However, it has persisted subconsciously, if not consciously, through each century up to and including the $21^{\text {st }}$. Biology became a separate science in the $19^{\text {th }}$ century. In the latter half of the $20^{\text {th }}$ century evolutionary biologists and philosophers of biology had continually argued for an autonomous biology distinct from the physical sciences (Ayala, 1968, 2000 ). Ernst Mayr, one of the $20^{\text {th }}$ century's leading evolutionary biologists, argued for the autonomy of biology in chapter 2 of a book published in 2004 (Mayr, 2004). Recently, Bhakti Niskama Shanta wrote at length on 'Why biology is beyond physical sciences' (Shanta, 2016). Mayr went further and stated that "it was not until the second half of the $20^{\text {th }}$ century that biology acquired dominance among the sciences" (Mayr, 2004). Such statements are unhelpful as 'dominance among the sciences' implies most significant in the universe and are rooted in an anthropocentric world view.

The main reason, given by philosophers, for biology being beyond the physical sciences is that physical sciences are reductionist, which is incorrect, while biology is holistic. If one truly believed in holism, as opposed to reductionism, science ought to be moving in the direction of integration and not autonomy. The insistence on autonomy is preventing development of an integrated holistic overview of all of science. Also, such autonomy prevents mind and consciousness being brought within the laws of physics. The evolutionary biologists cannot have it both ways. A biology that is an autonomous holistic science, separate from all other sciences, is a contradiction. In arguing for the autonomy of biology, they suggest that science in general is not holistic, but that biology is. Contrary to their assertions, physics ceased to be purely reductionist, at the beginning of the $20^{\text {th }}$ century when quantum mechanics became evident. Quantum mechanics provides a holistic view of the world that we have yet to fully understand. We still don't know what quantum mechanics is trying to tell us about nature.

If insistence on the autonomy of biology from the rest of the sciences had no consequences one might ignore this naïve intuition. However, the belief in the autonomy of biology is preventing the development of an integrated picture of how the key structures, particles and forces of the universe relate to each other. As such, the persistence of this variation of a pre-Copernican intuition (autonomy), be it conscious or subconscious, is unacceptable as it is preventing progress and for this reason must be eradicated. The fundamental flaw in the thinking of philosophers of biology is that they assume the physics we know, today, is all there is. Our minds continually jump to conclusions based on what we know and assume that all there is to know, is known. We are especially prone to jumping to conclusions that we were pre-disposed to having (e.g. anthropocentrism) as they lie suppressed in our subconscious from the past and have not been supplanted (Shtulman 2012). This is a common flaw with human thinking that has been addressed by psychologists such as Daniel Kahneman and others (Kahneman, 2011). It requires vigilance if we are to avoid it. As on innumerable previous occasions since the dawn of man it will soon become clear, yet again, that all of physics is not yet known. The usual incremental approach to biology has, so far, failed to deliver progress and a more holistic approach is needed to see how we might move forward. This progress will only happen if we are able to overcome the current adherence of the mainstream scientific community to pre-Copernican naïve intuitions about biology's autonomy from the rest of the physical sciences.

\section{Cell and inter-cell Communication}

The cell is a very complex and stable system in which many processes (e.g. transport, metabolism, growth and production of useful products and waste, regulation and internal and external defence, energy distribution, adaptation, replication, cell division, hierarchical organisation, environment monitoring etc.) must proceed in an orderly fashion over time. To achieve all this the cell needs a very sophisticated, secure, long range (over cellular distances), almost instantaneous communication system to transfer all the information needed to prevent a decent into chaos. This must occur on an ongoing basis for the lifetime of the structure to maintain stability. A complete understanding of a single cell is a long 
way off as it is a much more complex system than we have imagined so far. It took three billion of the four and a half billion years since the earth was formed to perfect the single cell which is, of itself, a measure of its complex communication system. It is not hard to imagine that in the time, before multicellular plants and animals evolved, this rudimentary communication system became more sophisticated and eventually became intracellular (eventually up to the scale of the mind) allowing the construction of specialised cellular materials and hence specialised structures for various functions of larger multicellular plants and animals. One of these would have been a rudimentary command and control centre that would, with time, evolve into what we now call the brain and mind. Given that we know so little about how 'global' information is communicated within or between cells it would be unwise to push suggested models (Goodman, 2015; 2016) for the mind and consciousness too far. After all the human brain contains tens of billions of multiply interconnected neurons and we do not possess a full understanding of how even one neuron works.

It has often been said that the brain/mind does not act like a computer as classically understood. This comes from years of experience with AI both from the life mimicking (cellular automata or evolutionary programming) or mind mimicking (artificial neural networks or logic based classical AI) perspectives. With $\mathrm{AI}$, we have never come close to mimicking what the brain can do, nor have we succeeded in creating an artificial consciousness. In neuroscience by contrast, a complete connectome of a tiny worm (Caenorhabditis elegans), which has only 302 nerve cells in the creature's 'brain' is less well understood after over 30 years of study than computer chips with billions of components and billions of inter-connections. This clearly points to the fact that the assumption that minds like computers are processing information by moving electric signals around complicated circuits cannot be correct except on the very basic level such as neurotransmission (i.e. the interface between brain and body).

\section{Quantum Mechanics in Biology}

The mind and consciousness appear to share quantum mechanical features such as holism and instantaneous thought (inspiration) analogous to quantum collapse per the Copenhagen interpretation of quantum mechanics. These similarities led to dozens of attempts (Tarlachi, 2010a) to develop a theory, during the 20 $20^{\text {th }}$ century, which linked the two fields, without success. The current belief is that the brain operates on a classical macroscopic scale (> 1 micron) and a quantum microscopic (subatomic, atomic and molecular) scale. There are residual 'fuzzy' quantum/classical effects up to short of 100 $\mathrm{nm}$ and no quantum mechanical effects above this scale. We currently think this because, like the philosophers of biology, we believe what we can see quantum mechanically is all there is. This is misguided and, as was stated previously, a common flaw with human thinking. The laws of quantum mechanics apply to all fundamental particles irrespective of type (quark or lepton), force operating (strong or electromagnetic) or associated force property (colour or charge). The common perception (Figure 2, Tarlachi, 2010b) that quantum mechanics is restricted to less than the 100 nanometer scale is an illusion that is solely due to the mass of the electron being so "big". A mass one million times smaller than the electron would allow, by comparison with quantum mechanics at the atomic and molecular level, quantum mechanics to operate over a range one million times bigger from $10^{-6} \mathrm{~m}$ up to $10^{-1} \mathrm{~m}$. (Coincidentally the scale associated with the mind). This relates to the fact that if you had no knowledge of a particles uncertainty in momentum it's uncertainty in position must be $>\mathrm{h} / 2 \pi \mathrm{mc}$ where $\mathrm{h}$ is Planck's constant, $\mathrm{c}$ is the speed of light and $\mathrm{m}$ is the mass of the particle. A mass one million times smaller has an uncertainty in position that is one million times greater. Such a particle mass is about to be measured. It is the electron neutrino. It took us till the start of the $20^{\text {th }}$ century to recognise the existence of holistic quantum mechanics at all and to be able to measure holistic quantum mechanical effects. The quantum mechanical effects associated with the electron neutrino will be a million times subtler, than those associated with the electron, making them much harder to measure and observe providing an explanation for why we have not noticed them before now. Finally, it appears that it is the uncertainty in position of the associated fundamental particle that determines the effective force range, and the range over which communication can occur, in each associated structure. These are the quark in the case of the nucleon and strong force, the electron in the case of the atom and the electromagnetic force, and as proposed since 1994 (Goodman, 1994) the 
electron neutrino in the case of the biological cell and the weak force. See Figure 1 below.

\begin{tabular}{|l|l|l|l|}
\hline \hline Fundamental Science & Nuclear Science & Chemistry & Biology \\
\hline Force dominating & Strong & Electro-magnetic & Weak \\
\hline $\begin{array}{l}\text { Self-organising System } \\
\text { uncertainty in particle } \\
\text { position }\end{array}$ & quarks (q) & &
\end{tabular}

Figure 1. How 'global' communication is achieved in the key selforganising systems found in nature

By analogy with the other key structures such as nucleons and atoms information transfer could be securely delivered over cellular distances via the electron neutrino whose uncertainty in position, like the quark in the nucleon and the electron in the atom, is of the same order as the size of the structure itself creating a 'long range' communication vehicle which could operate almost instantaneously in the cell. The rules of quantum mechanics as they apply to the neutrino and cell could then be used to construct, over time, an information or communication system to transfer all necessary information to maintain order, in the biological cell and prevent the constant threat of a decent into chaos.

The brain could then use quantum mechanical neutrino interactions between existing atomic nuclei (Goodman 2015) to create the mind where a 'global' communication and mental experience (consciousness) could take place. That mind would be physical but weigh next to nothing (but not nothing) making it difficult to detect. This provides Loewenstein's (Loewenstein, 1999) two requisites for quantum computation to be possible in the brain, i.e. insulation from the electromagnetic "cell sap" and intracellular continuity to allow for multicellular quantum coherent states allowing for consciousness to be mostly quantum mechanical in nature as has long been suspected. Separate from this the usual quantum chemical processes will still rule at the "local" atomic and molecular level and be responsible for all local properties of the cell including local information transfer. Local physical functions such as neurotransmission and all input/output information to and from the brain will also be of a chemical nature and occur through electromagnetic interactions. This separation of mind (through weak-neutrino interactions) and brain (through electromagnetic-electron interactions) should help us begin to bridge the "explanatory gap" that presently appears to exist between the 'mental' and 'physical' aspects of the brain.

Initially the focus should be on working toward a complete model for quantum information transfer within cells. This information system would weigh very little by comparison with the mass of the cell and, hence, would be difficult to observe. The next step would be to investigate what materials cells can make from the purely structural all the way up to advanced/smart materials that are used in the mind that can deliver consciousness. Biology displays innumerable examples of materials that are way more advanced and smarter (mind/brain) than any we can conceive. We do not fully understand either the construction or function of most of these. This is where the focus on smart/advanced materials should be.

\section{Quantum Mechanics as Information}

Since the 1970's quantum mechanics has been conceived of as information, mostly. As Christopher Fuchs puts it 'Quantum mechanics has always been about information; it is just that the physics community has forgotten this' (Fuchs 2003). The quantum state of a system is just an expression of subjective information. In the 1990's the distinguished theoretical physicist John Wheeler proposed that at a fundamental level all of physics can be described in terms of information. In 2014 a paper (Coles et al., 2014) showed that there was a deep connection between information theory and quantum mechanics by showing waveparticle duality corresponds precisely to the uncertainty principle in terms of the so-called minand max-entropies used in cryptography.

Inside the nucleon the quarks interact with each other and any associated nucleons, via the rules of quantum mechanics, to determine which of the large number of arrangement possibilities are allowable and which are not in the assembly of nucleons and nuclei. The same quantum mechanical rules for electrons determine which of the large number of arrangement possibilities are allowable and which are not in the assembly of atoms and molecules This constitutes information transfer via fundamental particles and associated structures about the 'state' of the system and is 
quantum communication at a very fundamental level. It is this continual information transfer over time that determines the stability of these structures. At a very basic level, it appears that quantum mechanics determines how all particles interact and how the structures they form communicate to maintain stability. This leads naturally to an understanding of the physical significance of quantum mechanics in nature as an alternative to the axiomatic approach used to date See Table 1 below.

Table 1. A physical interpretation of the significance of quantum mechanics in nature as an alternative to the more usual axiomatic approach

\begin{tabular}{|c|c|}
\hline $\begin{array}{c}\text { Quantum Mechanical } \\
\text { Axioms }\end{array}$ & Related Physical statements \\
\hline \multicolumn{2}{|l|}{$\begin{array}{l}\text { 1. Any physical quantum } \\
\text { system has a finite discrete set } \\
\text { of energy levels }\end{array}$} \\
\hline & $\begin{array}{l}\text { 1. Every self-organising system } \\
\text { (SOS) in nature is a quantum } \\
\text { mechanical system }\end{array}$ \\
\hline \multicolumn{2}{|l|}{$\begin{array}{l}\text { 2. The state of a system at any } \\
\text { time is a quantum } \\
\text { superposition of states }\end{array}$} \\
\hline & $\begin{array}{l}\text { 2. All communication/information } \\
\text { transfer across the entire SOS is } \\
\text { quantum mechanical in nature }\end{array}$ \\
\hline \multicolumn{2}{|l|}{$\begin{array}{l}\text { 3. Observation/Measurement } \\
\text { causes the systems state to } \\
\text { change }\end{array}$} \\
\hline & $\begin{array}{l}\text { 3. The physical part of the } \\
\text { communication system is the } \\
\text { associated particle. (with a mass } \\
\text { inversely proportional to system } \\
\text { size) }\end{array}$ \\
\hline $\begin{array}{l}\text { 4. Systems evolve via the } \\
\text { Schrodinger wave equation }\end{array}$ & \\
\hline
\end{tabular}

Finally, in relation to security no-one would argue that quantum theory also predicts the existence of quantum cryptography. It would be surprising if the cell did not make use of this quantum mechanical feature to survive.

\section{Recent Neutrino Mass findings}

The upper limit on the sum of the three neutrino masses is $<0.3 \mathrm{eV} / \mathrm{c}^{2}$ via cosmology (Goobar et al, 2006). This upper limit is model dependent. The lower limit is $>0.04 \mathrm{eV} / \mathrm{c}^{2}$ via solar oscillation experiments (Amsler et al., 2008). The KATRIN experiment in Karlsruhe, Germany has been fully operational since early June 2018. The non-result in the first six months of operation means that the upper limit on the mass of the electron neutrino, with a greater than $90 \%$ confidence level, is heading for less than $0.5 \mathrm{eV} / \mathrm{c}^{2}$ (Fig 26(b), Drexlin et al., 2013). This upper limit will soon lie in the range first predicted, some 30 years ago, and first published in 1994 (Goodman, 1994). That prediction did not attract much attention as it was some three hundred times below the upper limit of the day and at a time when most supporters of the Standard Model believed that the mass of the electron neutrino was zero. The nett effect was that measurement of the electron neutrino mass was some decades away and so it stimulated little interest. This non-result, from the KATRIN experiment, suggests that the mass of the electron neutrino lies below $0.5 \mathrm{eV} / \mathrm{c}^{2}$ making the quantum connection with biology, suggested in this and previous papers, more likely. Because it has been so difficult to measure and detect neutrinos, due to their tiny mass, our picture of how these neutrinos can interact, and how often, with nuclei at low energy is still uncertain. It is this interaction that is key to a 'global' quantum communication in the cell and is where our attention should turn next.

\section{Conclusions}

All the sciences have reductionist and holistic features. Biology is no exception and is a physical science that is not autonomous from the rest. Quantum mechanics is not restricted to the atomic and molecular scales. It has a crucial role in all structure at all scales through quantum communication and information transfer which creates stability and prevents the structure from returning to equilibrium and ending in a chaotic break up. Quantum mechanics is mostly information with an ever-smaller (never zero) physical part associated with the relevant fundamental particle that conveys information. The larger the self-organising system is the smaller the information carrying physical part becomes. It is the uncertainty in position of the fundamental particle that determines the size of the selforganising system. Just as the quark is the messenger in the nucleon and the electron is the messenger in the atom, the e-neutrino is the information transfer mechanism in the cell that is vital to cell stability. This gives a glimpse of the significance of quantum mechanics and what it has been trying to tell us about nature since the beginning of the $20^{\text {th }}$ century. Namely, all information transfer is quantum mechanical and the physical part of communication, in any selforganised system, is the associated fundamental particle and how it interacts with its associated system. After 30 years the mass of the electron neutrino is finally in the range predicted initially in 1988, implicating it in an information transfer role in Biology. 


\section{References}

Amsler C, Doser M, Antonelli M, Asner DM, Babu KS, Baer H, Band HR, Barnett RM, Bergren E, Beringer J, Bernardi G. Review of particle physics. Physics Letters, Section B: Nuclear, Elementary Particle and High-Energy Physics 2008; 667(1-5): 1-6.

Ayala FA. Biology as an autonomous science. American Scientist 1968; 56: 207-21.

Ayala FJ. Evolution and the Autonomy of Biology. AQUINASROME 2000; 43(2): 283-312.

Coles PJ, Kaniewski J, Wehner S. Equivalence of wave-particle duality to entropic uncertainty. Nature Communications 2014; 5: 5814

Drexlin G, Hannen V, Mertens S, Weinheimer C. Current direct neutrino mass experiments. Advances in High Energy Physics 2013;2013: 21.

Fuchs CA. Quantum mechanics as quantum information, mostly. Journal of Modern Optics 2003; 50(6-7): 987-1023.

Goobar A, Hannestad S, Mörtsell E, Tu H. The neutrino mass bound from WMAP 3 year data, the baryon acoustic peak, the SNLS supernovae and the Lyman- $\alpha$ forest. Journal of Cosmology and Astroparticle Physics 2006; 2006(06): 019.

Goodman M. Key self-organising systems. Proceedings of the 1st ICASSE 1994; 94: 118-25.

Goodman M. Toward linking material self-organization and the weak force. Speculations in Science and Technology 1997;2 0(1): 33-44.
Goodman M. A quantum theory of consciousness may require a paradigm shift in biology. Journal of Consciousness Exploration \& Research 2015; 6(1): 1-9.

Goodman M. Suppressed intuitions, large number coincidences, and a mathematical foundation for life and consciousness. NeuroQuantology 2016 Jan 6;14(1): 62-67.

Goodman M. The mainstream scientific community is open to incremental but not holistic ideas. Neuroquantology 2017; 15(2): 215-16.

Kahneman D. Thinking, Fast and Slow. Oxford: Penguin, 2012: 85-88.

Loewenstein W. The Touchstone of Life. Oxford: Penguin, 1999: 400-06.

Mayr E. What makes Biology unique? Cambridge: University Press, 2004: 21-38.

Shanta BN, Muni BV. Why biology is beyond physical sciences?. Advances in Life Sciences 2016; 6: (1): 13-30.

Shtulman A, Valcarcel J. Scientific knowledge suppresses but does not supplant earlier intuitions. Cognition 2012; 124(2): 209-15.

Tarlaci S. A historical view of the relation between quantum mechanics and the brain: a NeuroQuantologic perspective. NeuroQuantology 2010a; 8(2): 120-36.

Tarlacl S. Why we need quantum physics for cognitive neuroscience. NeuroQuantology 2010b; 8(1): 66-76. 